\title{
Perils of pandemic waves in COVID-19
}

This article was published on $2 \mathrm{Feb}$ 2021 at www.hkmj.org.

\author{
Samuel SY Wang ${ }^{1}$, Bmed, MD, Winnie ZY Teo ${ }^{1,2}$, MB BcH BAO, MRCP
}

${ }^{1}$ Fast Program, Alexandra Hospital, National University Hospital System, Singapore

${ }^{2}$ Department of Haematology-Oncology, National Cancer Institute Singapore (NCIS), National University Health System, Singapore

*Corresponding author: samuel.wang@mohh.com.sg

Hong Kong Med J 2021;27:67-9

https://doi.org/10.12809/hkmj208723

The coronavirus disease 2019 (COVID-19) pandemic is a pressing global health challenge in $2020 .{ }^{1}$ Globalisation and improvements to air travel have fuelled the global spread of severe acute respiratory syndrome coronavirus 2 (SARS-CoV-2) that causes COVID-19. At the time of writing, treatment methods for COVID-19 are largely supportive owing to the lack of effective antiviral medications or vaccination. Therefore, tackling the pandemic involves breaking the chain of infection through testing, quarantine and social distancing. ${ }^{2}$ Based on our current experience with dealing with SARS-CoV-2 patterns of infection and new resurgence in cases due to various vulnerable populations, ${ }^{3}$ we would like to highlight potential populations that might benefit from closer surveillance and asymptomatic pre-emptive testing. ${ }^{4}$ This is to prevent new waves of COVID-19 infections.

Marginalised and economically vulnerable populations can potentially become infection clusters. ${ }^{5}$ An example would be migrant shift workers living in unsanitary and overcrowded accommodations. ${ }^{5}$ Among this demographic, quarantine and social distancing policies will have the greatest economic impact. As their work is unstable and with minimal worker compensation benefits, tighter social distancing policies often lead to job and income losses. Therefore, workers are often caught between sacrificing their income/economic livelihood and public health policy. Hence, they may continue working despite public health policies, which compromises social distancing effectiveness. They may also underreport symptoms or not seek medical help fearing work cessation and job/income loss. With tighter social distancing policies causing businesses to close, these populations might migrate back to their home countries/home towns due to retrenchment or to avoid being separated from their families during the lockdown, possibly transmitting infections and overwhelming rural health services..$^{5,6}$ This was the fear that Indian and Indonesian governments had when they increased lockdown measures to stem the pandemic. ${ }^{7}$

Another issue is the existing poor health literacy, education level, cultural and language barriers. This may lead to difficulty in communicating public healthcare policies. Additionally, communication barriers due to language may impede direct clinical care. The communication barriers may also generate fear of discrimination which may alter healthseeking behaviour by these populations. Another potential problem is that such workers are foreigners and are potentially undocumented migrants leading them being overlooked by healthcare planners. ${ }^{8}$ Finally, due to their poor hygiene and cramped living conditions, SARS-CoV-2 infections will spread more readily. ${ }^{8}$ These factors create a perfect storm for seeding of occult infections, triggering an explosion of new infection clusters, and precipitating new pandemic waves as seen in Singapore.

Therefore, to address these challenges, multiple public health and economic measures must be undertaken to support this often-marginalised population. Firstly, would be to establish a suitable housing environment to improve personal hygiene and overcrowding. Next would be to provide financial/economic benefits to encourage social distancing and lockdown compliance. Raising healthcare literacy through culturally and language appropriate communication of healthcare policies would be especially needed for this population.

The elderly, frail, and immunocompromised healthcare residents are another vulnerable population. These populations are vulnerable to infections and subsequent deterioration due to their impaired immune system, this may also translate to higher viral loads and higher transmission risk. ${ }^{3,9}$ Also due to their frailty and dependence on carers they face greater difficulty practising effective social distancing leading them to transmit or acquire SARS-CoV-2 infections. This can cause severe outbreaks in age care facilities, hospices and elder care centres, ${ }^{10}$ leading to consumption of acute healthcare resources such as intensive care unit beds due to their high risk for deterioration. For the elderly people who are living in the community, some practical real-life examples of social distancing measures can be seen globally which minimises the elderly people's exposure with the rest of the community. Special shopping timings are created for elderly/vulnerable population shoppers so that they do not have to mix with the rest of the community. ${ }^{11}$ Contactless delivery services involve items being placed at the door of the recipient's home and the 
delivery person ringing the doorbell to alert the recipient and then standing at a safe distance of $1 \mathrm{~m}$ while waiting for the recipient to pick up the item. ${ }^{12}$ This process would minimise physical contact between the delivery person and the recipient. ${ }^{12}$ This service enables food and essentials to be supplied to the elderly people who are self-isolating. ${ }^{12}$ If the elderly people are institutionalised in a healthcare facility the situation is much more difficult. This would involve stricter restrictions on visitor policies limiting to one regular visitor per patient and only if the patient is imminently dying will the restriction be lifted. ${ }^{13}$

Another group to consider are young paediatric populations, which are less symptomatic when infected and able to recover faster. ${ }^{14}$ This intuitively is a beneficial scenario for patients, but in terms of population health, it may complicate contact tracing and social isolation. ${ }^{14}$ Being dependent on their parents/carers, they are similar to elderly patients in being unable to practise effective social distancing. Additionally, children may have less adequate personal hygiene and may not understand the public health implications. They may also transmit infections among the elderly people as senior citizens are often involved in child care. ${ }^{15,16} \mathrm{~A}$ silver lining is the potential reduced risk for clinical deterioration, hence reduced consumption of acute healthcare resources. This population might benefit from closer supervision from their parents and temporary school closures.

Similar to paediatric populations would be youths who are also less symptomatic when infected and recover faster. ${ }^{14}$ However, due to self-perceived low health risk from COVID-19, there is poorer compliance with social distancing which may sustain community-based infections. However, youths are likely to be severely impacted socio-economically by COVID-19, as they may be graduating into a pandemic and a post-pandemic recession. ${ }^{17}$ Furthermore, many youths may encounter job losses or reduced income because of social distancing measures..$^{17}$ Other important life events such as major academic examinations, and university graduations may be delayed. Thus, awareness of such issues should be highlighted to the youth to increase social distancing compliance. This population would require frequent communication and enforcement of healthcare policies. Additionally, post pandemic this population would require social, financial and economic policies to reduce the socio-economic fallout from unemployment and lost educational opportunities. ${ }^{17}$

Similar to migrant workers, sex workers are another vulnerable population as social distancing reduces demand for their services leading to income loss. Thus, they may be pressured to aggressively seek out clients to compensate for lost income thereby compromising social distancing measures. Again, due to unstable/undeclared income sources, they may be neglected by economic assistance policies. Also, due to their work and the risk of discrimination they may be less inclined to seek healthcare services. Sex workers often have unstable and crowded housing therefore limiting social distancing opportunities. Additionally, despite SARS-CoV-2 being predominantly spread through droplets not all potential transmission routes have been fully studied. Currently, vaginal sexual transmission is unlikely due to lack of SARS-CoV-2 in vaginal swabs; however, sexual transmission via the faeco-oral route may be an unidentified transmission route. ${ }^{18}$ Vertical transmission of SARS-CoV-2 has also been suspected between mothers and neonates, though the results have not been confirmed. ${ }^{19}$ Similar policies to the migrant workers should be provided to sex workers such as improving housing and financial/economic benefits to encourage social distancing/lockdown compliance.

This paper hopes to raise awareness of possible waves of infection arising from certain populations and the health/socio-economic impact that the COVID-19 pandemic may have on them. Through awareness of these populations, national taskforces and healthcare workers in the field will be better equipped to assist these populations while containing the COVID-19 pandemic.

\section{Author contributions}

All authors were involved in the concept or design, acquisition of data, analysis or interpretation of data, drafting of the manuscript, and critical revision of the manuscript for important intellectual content. All authors had full access to the data, contributed to the study, approved the final version for publication, and take responsibility for its accuracy and integrity.

\section{Conflicts of interest}

The authors have no conflicts of interest to disclose.

\section{Funding/support}

This commentary received no specific grant from any funding agency in the public, commercial, or not-for-profit sectors.

\section{References}

1. Lai CC, Shih TP, Ko WC, Tang HJ, Hsueh PR. Severe acute respiratory syndrome coronavirus 2 (SARS-CoV-2) and coronavirus disease-2019 (COVID-19): The epidemic and the challenges. Int J Antimicrob Agents 2020;55:105924.

2. Anderson RM, Heesterbeek $H$, Klinkenberg D, Hollingsworth TD. How will country-based mitigation measures influence the course of the COVID-19 epidemic? Lancet 2020;395:931-4.

3. CDC COVID-19 Response Team. Severe outcomes among patients with coronavirus disease 2019 (COVID-19)United States, February 12-March 16, 2020. MMWR Morb Mortal Wkly Rep 2020;69:343-6. 
4. Bai Y, Yao L, Wei T, et al. Presumed asymptomatic carrier transmission of COVID-19. JAMA 2020;323:1406-7.

5. Kluge HH, Jakab Z, Bartovic J, D'Anna V, Severoni S. Refugee and migrant health in the COVID-19 response. Lancet 2020;395:1237-9.

6. Chen S, Yang J, Yang W, Wang C, Bärnighausen T. COVID-19 control in China during mass population movements at New Year. Lancet 2020;395:764-6.

7. Al Dahdah M, Ferry M, Guérin I, Venkatasubramanian G. The Covid-19 crisis in India. 2020. Available from: https:// booksandideas.net/The-Covid-19-Crisis-in-India.html. Accessed 12 May 2020.

8. Meyer JP, Franco-Paredes C, Parmar P, Yasin F, Gartland M. COVID-19 and the coming epidemic in US immigration detention centres. Lancet Infect Dis 2020;20:646-8.

9. Chen Y, Li L. SARS-CoV-2: virus dynamics and host response. Lancet Infect Dis 2020;20:515-6.

10. Gardner W, States D, Bagley N. The coronavirus and the risks to the elderly in long-term care. J Aging Soc Policy 2020;32:310-5

11. Nicola M, Alsafi Z, Sohrabi C, et al. The socio-economic implications of the coronavirus pandemic (COVID-19): A review. Int J Surg 2020;78:185-93.

12. Nguyen TH, Vu DC. Food delivery service during social distancing: proactively preventing or potentially spreading coronavirus disease-2019? Disaster Med Public Health Prep 2020;14:e9-e10.

13. Hsu YC, Liu YA, Lin MH, et al. Visiting policies of hospice wards during the COVID-19 pandemic: an environmental scan in Taiwan. Int J Environ Res Public Health 2020;17:2857.

14. Dong Y, Mo X, Hu Y, et al. Epidemiology of COVID-19 among children in China. Pediatrics. 2020;145:e20200702.

15. Li C, Ji F, Wang L, et al. Asymptomatic and human-tohuman transmission of SARS-CoV-2 in a 2-family cluster Xuzhou, China. Emerg Infect Dis 2020;26:1626-8.

16. Liu J, Liao X, Qian S, et al. Community transmission of severe acute respiratory syndrome coronavirus 2, Shenzhen, China, 2020. Emerg Infect Dis 2020;26:13203.

17. McKee M, Stuckler D. If the world fails to protect the economy, COVID-19 will damage health not just now but also in the future. Nat Med 2020;26:640-2.

18. Patrì A, Gallo L, Guarino M, Fabbrocini G. Sexual transmission of severe acute respiratory syndrome coronavirus (SARS-CoV-2): a new possible route of infection? J Am Acad Dermatol 2020;82:e227.

19. Dong L, Tian J, He S, et al. Possible vertical transmission of SARS-CoV-2 from an infected mother to her newborn. JAMA 2020;323:1846-80. 